\title{
Comprehending the Pricing Decision of Online Car-Hailing Services in China: Price Regulation Vs Entry Limitation
}

\author{
Jie Yang ${ }^{\mathrm{a}, 1}$ Daozhi Zhao ${ }^{\mathrm{b}}$ Zeyu Wang $^{\mathrm{c}}$ Wanying Jiang ${ }^{\mathrm{d}}$ \\ ${ }^{a}$ School of Social Science, Tsinghua University, 30 Shuangqing Road, Haidian District, \\ Beijing, 100084, China \\ ${ }^{\mathrm{b}}$ College of Management and Economics, Tianjin University, 92 Weijin Road, Nankai \\ District, Tianjin, 300072, China \\ ${ }^{c}$ Department of Computer Engineering, Texas A\&M University, 400 Bizzel St, College Station, \\ $T X, 77840$, USA \\ d School Of Psychology, Northwest Normal University, 967 Anningxi Road, Anning District, \\ Lanzhou, 730070, China
}

\begin{abstract}
Online car-hailing service has had an exponential growth in recent years, and poses a substantial threat to taxi service. Yet how to regulate online car-hailing service has not been adequately studied. Based on the regulation of tradition taxi service, price regulation and entry limitation are used to regulate online car-hailing service in this paper. Moreover, we consider two types of online car-hailing service, i.e., high-end service(e.g. UberBlack) and low-end service (e.g., UberX), according to the perceived service level. Then, the optimal platform's price is formulated. The result shows that the price regulation are likely to increase the optimal platform price, depending on the service type and the taxi price. When the platform offers low-end service and the taxi price is low, the optimal platform price does not change. In contrast, the entry limitation reduces the optimal platform's price when it achieves the regulation target.
\end{abstract}

Keywords. Sharing economy, online car-hailing service,taxi service, price regulation, entry limitation

\section{Introduction}

Facilitated by the information technology, many business operations are developing in online mode [1]. An example of such business models is the online car-hailing service, which enables riders to request rides online from independent drivers nearby [2]. It collects drivers offering ride services to the wider public based on coordination through a platform for centralized dispatch[3]. The popularity of online car-hailing service relies not only on advanced technology, but also on a lower price[4], higher flexibility[5], and environment friendly [6-7]. Therefore, the emergence of online carhailing service presents a tremendous disadvantage to traditional taxi service. In China,

${ }^{1}$ Corresponding Author, Jie Yang, School of Social Science, Tsinghua University, 30 Shuangqing Road, Haidian District, Beijing,100084, China. E-mail: nemoyj1989@126.com. 
the transaction volume of online car-hailing service in 2017 exceeded 200 billion with a potential increase [8].

The rapid development of online car-hailing platforms leads to the decline of taxi demand [9]. Correspondingly, the profit of taxi service is reduced. Taxi drivers are unsatisfied with the entry of online car-hailing service and claim that the government imposed different rules on the entrant than they must follow [10]. Thus, online carhailing service has brought out emerging issues such as regulation. However, the research on the regulation policy is very few. This paper, motivated by the growth and the new rules of online car-hailing service, is focused on the two regulation policies, i.e., price regulation and entry limitation, according to game theoretic method of modeling. And we also characterize the two types of online car-hailing services by different perceived service levels compared to that of taxi service, i.e., high-end service and low-end service. On one hand, we aim to theoretically discuss about the impact of two regulation policies on the price strategy of the platform. On the other hand, we also hope to provide some suggestions about regulation of online car-hailing service for the government.

Our main findings are as follows. Our analysis indicates that the price regulation increases the optimal platform's price when the platform offers high-end service, whereas when the platform offers low-end service, the optimal platform's price does not change with and without regulation under certain circumstance. It is related to the taxi service price and the perceived service level. The entry limitation reduces the optimal price of online car-hailing service. The result shows that entry limitation benefits not only taxi service but also riders.

The remainder of this paper is organized as follows. In Section 2, we provide a review of related literature. In Section 3, we develop the model and analyze the model. In Section 4, we conduct the comparison analysis. We conclude the paper in Section 5.

\section{Literature review}

This paper draws on three different research streams: studies about the taxi service, researches focused on the ridesharing service and the researches on the regulation of the transportation services.

[11] develops the first taxi model in an aggregated way, exploring the problem of the optimal price and the optimal service quality. Based on the model proposed by [11], [12] builds a model of taxi industry and determines equilibrium output and capacity in different market configurations. Then, researchers have been made to better understand and characterize the mechanism of how taxi demand and supply are equilibrated, and the impact of different market configurations; moreover, researchers have been incorporated the spatial structure of taxi services into models [13-16]. Since online carhailing service resembles taxi service[9], we follow this stream to adopt the similar assumptions.

There is growing research that focuses on online car-hailing service, especially the platform pricing decision. Several papers study the platform price in static price policy or dynamic price policy [17-19]. Related research consider the riders and drivers behavior, such as delay sensitivity[20-21] and drivers idle-time sensitivity [22]. [17] suggests that dynamic policy can ensure a high service level in high demand periods. [23] argues that surge pricing can be a useful method in places where demand is even 
lower than supply. For an extensive review on the research online car-hailing service, we refer readers to[24-25].

More relevant to this paper are literatures investigating the regulation of transportation service, especially the regulation of the ridesharing service. The regulation of taxi service focuses on price, quantity and quality [26-27]. [28] studies the impact of Uber on taxi service in the US and claims that cities with the taxicab medallion system should reduce the cost of taxi service when Uber entries the market. [29] considers the competition between two ridesharing service platforms and explores the effect of different regulation modes, such as price adjustments regulation and the joint with official platforms. Similarly, considering different regulation policies, [30] analyzes a two-period dynamic game to evaluate several performance measures such as consumer welfare and social welfare. Distinct from [29] and [30], we focus on the competition between online car-hailing service and taxi service, and we also consider two types of online car-hailing service according to the service experience. In addition, we attempted to compare the platform's price decision with different regulation polices, i.e., price regulation and entry limitation.

\section{Model}

\subsection{Monopoly model}

The ride market considered in this paper includes taxi service and online car-hailing service. Other ride services, such as tubes and buses, are considered as outside options. Taxis play a significant paratransit role to other public transportation modes because of its flexibility and personalized service capabilities [31]. Taxi service was free of competition before the entry of online car-hailing service. Thus, we first analyze a basic riders' choice model in a market with taxi service only. Riders are heterogeneous and differ in their perceived value of ride services. Let $v$, which is a random number, denote how much a rider values taxi service. We assume that $v$ follows uniform distribution, $U(0, V)$, and the total market size is normalized to one.

Ride service market is a dynamic time-varying system. We split time horizon (e.g., a whole day) into a set of periods to deal with such variances. When the duration of each period is sufficiently short, the market can be regarded as uniform in each period [32-33]. Note that we focus on the rider choice in one period excluding peak period and congestion period. We do not consider the case that demand exceeds supply. Moreover, Similar to [11], the trip length and the speed en route are assumed to remain constant in a single period within a geographic area. Therefore, the total cost of one trip can be considered as the service price [34]. The price of taxi service is strictly regulated by the government [35], who considers the public transport capacity and the volume of the road resource. Let $\tilde{p}_{T}$ represent the given price of taxi service. We assume that riders are rational, that is, they make the decision to maximize their utilities. The utility of using taxi service is given by $U_{T}=v-\tilde{p}_{T}$. Suppose the utility is set to be 0 when the riders take outside options without loss of generality. Clearly, riders who gain positive utility will choose taxi service. Therefore, the demand of taxi service is $\lambda_{T}=\operatorname{Pr}\left(U_{T} \geq 0\right)=1-\frac{\tilde{p}_{T}}{V}$. 


\subsection{Competition model without regulation}

The online car-hailing platform, such as Uber and Didi, characterized by its innovative mobile internet technology and various service types, has experienced exponential growth in recent years. The platform matches riders with independent drivers in real time by smartphone applications [2]. Different from taxi service, the price of online car-haling service is determined by the platform [35]. The platforms usually adopt low price strategy to attract riders [4]. Moreover, the platforms provide different services, i.e., high-end service (i.e., UberBLACK) and low-end service (i.e., UberX), according to the perceived service experience to better satisfy the riders' heterogeneous needs. In the competitive market, we assume that online car-hailing service is vertically differentiated with respect to taxi service. Let $\alpha$ reflect the riders' differential preference. The rider value online car-hailing service as $\alpha v$. Specifically, we assume that the perceived service level of high-end service is higher than that of taxi service, i.e., $\alpha>1$. And the perceived service level of low-end service is lower than that of taxi service, i.e., $0<\alpha<1$.

Let $p_{O}$ represent the price of online car-hailing service. The riders' utility of choosing online car-hailing service is $U_{O}\left(\alpha v, p_{O}\right)=\alpha v-p_{O}$. Similar to Section 3.1, either taxi service or online car-hailing service that offers a rider greater positive utility will be chosen by the rider. Given the prices of taxi service and online car-hailing service, the demand of both services, i.e., $\lambda_{T}$ and $\lambda_{O}$, is shown in Table 1 . Note that $p_{O H}$ and $p_{O L}$ denote the price of high-end service and low-end service, respectively.

Table 1 Demand of taxi service and online car-hailing service under different scenarios

\begin{tabular}{c|c|c|c}
\hline Scenario & Condition & $\lambda_{T}$ & $\lambda_{O}$ \\
\hline \multirow{5}{*}{$\alpha>1$} & $0<p_{O H} \leq \alpha \tilde{p}_{T}$ & 0 & $1-\frac{p_{O H}}{\alpha V}$ \\
\cline { 2 - 4 } & $\alpha p_{T}<p_{O H} \leq \tilde{p}_{T}+\bar{\alpha} V$ & $\frac{p_{O H}-\alpha \tilde{p}_{T}}{\bar{\alpha} V}$ & $1-\frac{p_{O H}-\tilde{p}_{T}}{\bar{\alpha} V}$ \\
\cline { 2 - 4 } $0<\alpha<1$ & $p_{O H}>\tilde{p}_{T}+\bar{\alpha} V$ & $1-\frac{\tilde{p}_{T}}{V}$ & 0 \\
\hline \multirow{5}{*}{$0<p_{O L}<\alpha \tilde{p}_{T}+\bar{\alpha} V$} & 0 & $1-\frac{p_{O L}}{\alpha V}$ \\
\cline { 2 - 4 } & $\alpha \tilde{p}_{T}+\bar{\alpha} V \leq p_{O L}<\alpha$ & $1+\frac{\tilde{p}_{T}-p_{O L}}{\bar{\alpha} V}$ & $\bar{\alpha} V$ \\
\cline { 2 - 4 } & $p_{O L} \geq \alpha \tilde{p}_{T}$ & $1-\frac{p_{O L}}{\alpha}$ & 0 \\
\hline
\end{tabular}

The platform's profit is given by $\pi_{O}\left(p_{O}\right)=\lambda_{O}\left(p_{O}-c_{O}\right)$, where $c_{O}$ represents the operation cost. Given the price of taxi service, the optimal prices of high-end service and low-end service without regulation are derived as $p_{N H}^{*}=\frac{\tilde{p}_{T}+c_{O H}+\bar{\alpha} V}{2}$ and $p_{N L}^{*}=\frac{\alpha \tilde{p}_{T}+c_{O L}}{2}$, where $c_{O H}\left(c_{O L}\right)$ is the operation costs of high-end (low-end) service. 


\subsection{Competition model with regulation}

The rapid development of online car-hailing platforms leads to the decline of taxi demand due to the low price and shorter wait times [9,36]. Correspondingly, the profit of taxi service is reduced. According to [37], taxi drivers have reference-dependent preferences around an earned income target. Since the taxi price is fixed in this paper, taxi drivers have reference-dependent preferences around a demand target. A reference point divides outcomes into gains or losses, thus creating a qualitative difference in the valuation of outcomes slightly above or below that reference point [38]. [39] proposes that it is natural to assume that the reference group is the set of agents playing against each other. Therefore, we assume taxi drivers choose the demand of online car-hailing service as the reference point of demand. If the regulation can ensure the taxi demand satisfies the reference point of demand, i.e., $\lambda_{T} \geq \lambda_{O}$, taxi drivers will accept the entry of online car-hailing service. Since online car-hailing service resembles taxi service [9] and regulation restrictions of taxi service include price regulation and entry limitation, we consider these two ways to ensure the coexistence of two services.

- Price regulation

Different from the case without regulation, the platform should consider the demand baseline of taxi drivers. Therefore, with price regulation, the price decision model of the platform is

$$
\begin{array}{ll}
\text { Max } & \pi_{O}\left(p_{O}\right)=\lambda_{O}\left(p_{O}-c_{O}\right) \\
\text { s.t. } & \lambda_{O} \leq \lambda_{T}
\end{array}
$$

Proposition 2. (a). The price of high-end service with price regulation should be in the interval $\left[\frac{\bar{\alpha} V+(2+\bar{\alpha}) \tilde{p}_{T}}{2}, \tilde{p}_{T}+\bar{\alpha} V\right)$. Moreover, the optimal price of high-end service with price regulation is $p_{P H}^{*}=\frac{\bar{\alpha} V+(2+\bar{\alpha}) \tilde{p}_{T}}{2}$,if $c_{T}<\tilde{p}_{T}<V ;(\mathbf{b})$. The price of low-end service with price regulation should be in the interval $\left[\frac{2 \alpha \tilde{p}_{T}+\alpha \bar{\alpha} V}{(\alpha+1)}, \alpha \tilde{p}_{T}\right)$. Moreover, the optimal price of low-end service with price regulation, $p_{P L}^{*}$, is shown in Table 2 .

Table 2 The optimal price of low-end service with price regulation

\begin{tabular}{ccc}
\hline Condition of $\alpha$ & Condition of $\tilde{p}_{T}$ & $p_{P L}^{*}$ \\
\hline$\alpha \in(0, \tau]$ & $\tilde{p}_{T} \in\left(\max \left\{c_{T}, \frac{c_{O L}}{\alpha}\right\}, \frac{\left(2 \alpha \bar{\alpha} V-c_{O L}(\alpha+1)\right)}{\alpha(\alpha-3)}\right]$ & $\frac{\alpha \tilde{p}_{T}+c_{O L}}{2}$ \\
$\alpha \in(0, \tau]$ & $\tilde{p}_{T} \in\left(\frac{\left(2 \alpha \bar{\alpha} V-c_{O L}(\alpha+1)\right)}{\alpha(\alpha-3)}, V\right)$ & $\frac{2 \alpha \tilde{p}_{T}+\alpha \bar{\alpha} V}{\alpha+1}$ \\
$\alpha \in(\tau, 1)$ & $\tilde{p}_{T} \in\left(c_{T}, V\right)$ & $\frac{2 \alpha \tilde{p}_{T}+\alpha \bar{\alpha} V}{\alpha+1}$ \\
\hline
\end{tabular}

Note that $\tau=\frac{c_{O L}+2 V-3 c_{T}+\sqrt{\left(c_{O L}\right)^{2}+\left(3 c_{T}-2 V\right)^{2}+2 c_{O L}\left(6 V-5 c_{T}\right)}}{2\left(2 V-c_{T}\right)}$. 
Proposition 2(a) indicates the price regulation always raises the price of high-end service, which is consistent with the result in the practice. The platform increases its price after the introduction of the new rules. Although online car-hailing service offers the service with a higher perceived service level, extravagant price compels riders who are indifferent between choosing taxi service or online car-hailing service in the absent of regulation to switch to taxi service. Proposition 2(b) indicates that under some circumstances, the optimal price of low-end service does not change with price regulation. Otherwise, the price regulation will raise the optimal price of low-end service. The condition is related to the taxi price and perceived service level coefficient, $\alpha$.

- $\quad$ Entry limitation

This paper focuses on the rider choice in one period excluding peak period and congestion period. Thus, the number of service providers can meet the demand as [11] does. The perceived service experience is based on the quality of drivers and convenience. However, considering the entry limitation, the number of service providers of online car-hailing service is reduced due to the restriction of the drivers' household registration and vehicle model. As a result, it increases the wait times of online car-hailing service. According to the data released by Didi, after the new rules launches, the difficulty of hailing a car at the high peak in Beijing, Shanghai, Guangzhou and Shenzhen in 2017 increases by $12.4 \%, 17.7 \%, 13.2 \%$ and $22.5 \%$, comparing with the data of the same period in the previous year [40]. Thus, we assume the perceived service experience of online car-hailing service is reduced due to the curtailment of the service supply by entry limitation. Similar to [41], we also use $\beta$ $(0<\beta<1)$ to denote the matching ratio of demand. It can also be referred as the regulation intensity. Then the utility of riders under entry limitation is modeled as $U_{O}^{E}=\alpha \beta v-p_{O}$. The price decision model of the platform is the same with Equation 1.

When the platform provides high-end service and the entry limitation is relatively loose $\left(\frac{1}{\alpha}<\beta<1\right)$, such as the new rules in Ji'nan which has no definite restrictions on the driver's household registration and vehicle models, the optimal platform's price is similar to that in the price regulation. The feasible interval of the price of high-end service with entry limitation is $p_{E H} \in\left[\frac{(\alpha \beta-1) V+(\alpha \beta+1) \tilde{p}_{T}}{2},(\alpha \beta-1) V+\tilde{p}_{T}\right]$. The optimal price of high-end service with entry limitation is $p_{E H}^{*}=\frac{(\alpha \beta-1) V+(\alpha \beta+1) \tilde{p}_{T}}{2}$, if $c_{T}<\tilde{p}_{T}<V$.

If the entry limitation is so stringent that the perceived service experience of highend services is reduced dramatically to be less than 1 , i.e., $0<\beta \leq \frac{1}{\alpha}$. The optimal price of high-end service is shown in Table 3.

When the platform offers low-end service, the perceived experience of online carhailing service is reduced from $\alpha$ to $\alpha \beta \quad(0<\beta<1)$ due to the entry limitation proposed by the government. The optimal price of low-end service with entry limitation is similar to that in the price regulation, substituting $\alpha$ with $\alpha \beta$. 
Table 3 The optimal price of high-end service with entry limitation when $0<\beta \leq \frac{1}{\alpha}$

\begin{tabular}{ccc}
\hline $\begin{array}{c}\text { Condition of } \\
\beta\end{array}$ & Condition of $\tilde{p}_{T}$ & $p_{E H}^{*}$ \\
\hline$\beta \in\left(0, \frac{\tau}{\alpha}\right]$ & $\tilde{p}_{T} \in\left(\max \left\{c_{T}, \frac{c_{O H}}{\alpha \beta}\right\}, \frac{\left(2 \alpha \beta(\alpha \beta-1) V-c_{O H}(\alpha \beta+1)\right)}{\alpha \beta(\alpha \beta-3)}\right]$ & $\frac{\alpha \beta \tilde{p}_{T}+c_{O H}}{2}$ \\
$\beta \in\left(0, \frac{\tau}{\alpha}\right]$ & $\tilde{p}_{T} \in\left(\frac{\left(2 \alpha \beta(\alpha \beta-1) V-c_{O H}(\alpha \beta+1)\right)}{\alpha \beta(\alpha \beta-3)}, V\right)$ & $\frac{2 \alpha \beta \tilde{p}_{T}+\alpha \beta(\alpha \beta-1) V}{\alpha \beta+1}$ \\
$\beta \in\left(\frac{\tau}{\alpha}, 1\right)$ & $c_{T}<\tilde{p}_{T}<V$ & $\frac{2 \alpha \beta \tilde{p}_{T}+\alpha \beta(\alpha \beta-1) V}{\alpha \beta+1}$
\end{tabular}

\section{Comparison analysis}

The comparison of the optimal platform's price and the demand of taxi service between the price regulation and the entry limitation when the platform provides high-end service is shown in Table 4.

Table 4 The comparison result of the optimal price and the demand between two regulation policies when $\alpha>1$

\begin{tabular}{cccc}
\hline $\begin{array}{c}\text { Regulation } \\
\text { intensity }\end{array}$ & $\tilde{p}_{T}$ & $\begin{array}{c}\text { The comparison of } \\
\text { optimal price }\end{array}$ & $\begin{array}{c}\text { The comparison of } \\
\text { taxi service's demand }\end{array}$ \\
\hline$\frac{1}{\alpha}<\beta<1$ & $\tilde{p}_{T} \in\left(c_{T}, \frac{\bar{\alpha} V+c_{O H}}{2 \alpha-1}\right)$ & $p_{E H}^{*}<p_{N H}^{*}<p_{P H}^{*}$ & $\lambda_{T}^{E}=\lambda_{T}^{P}>\lambda_{T}^{N}$ \\
$\frac{1}{\alpha}<\beta<1$ & $\tilde{p}_{T} \in\left(\frac{\bar{\alpha} V+c_{O H}}{2 \alpha-1}, V\right)$ & $p_{E H}^{*}<p_{P H}^{*}$ & $\lambda_{T}^{E}=\lambda_{T}^{P}$ \\
$\frac{\tau}{\alpha}<\beta<\frac{1}{\alpha}$ & $\tilde{p}_{T} \in\left(c_{T}, V\right)$ & $p_{E H}^{*}<p_{N H}^{*}<p_{P H}^{*}$ & $\lambda_{T}^{E}>\lambda_{T}^{P}>\lambda_{T}^{N}$ \\
$0<\beta<\frac{\tau}{\alpha}$ & $\tilde{p}_{T} \in\left(\max \left\{c_{T}, \frac{c_{O H}}{\alpha \beta}\right\}, V\right)$ & $p_{E H}^{*}<p_{N H}^{*}<p_{P H}^{*}$ & $\lambda_{T}^{E}>\lambda_{T}^{P}>\lambda_{T}^{N}$ \\
\hline
\end{tabular}

Note that $\lambda_{T}^{N}, \lambda_{T}^{P}$ and $\lambda_{T}^{E}$ represent the taxi demand in the market without the regulation, with price regulation and with entry limitation.

From Table 4, the optimal price with price regulation is the highest, and the optimal price with entry limitation is the lowest. This result contradicts the fact that the new rules focused on entry limitation increase the price of online car-hailing service. In this paper, we consider the impact of entry limitation on the riders' utility, which will reduce the demand. Whereas the demand in the practice usually is unchanged or change only slightly when the service supply decreases. Note that the reduction of drivers influences the perceived service experience of riders. For example, riders may wait for a longer time for the car's arrival. Therefore, the platform attracts riders by reducing the price. The entry limitation achieves the coexistence of the two services and enables riders to enjoy a higher perceived service experience with a lower price simultaneously. 
Comparing the optimal platform's prices with price regulation and entry limitation when the platform offers low-end service, we obtain the Proposition 3.

Proposition 3 When the regulation intensity is sufficiently stringent $\left(0<\beta<\frac{\tau}{\alpha}\right)$, there exists $p^{1} \in\left(\max \left\{c_{T}, \frac{c_{O L}}{\alpha \beta}\right\}, V\right)$ such that $p_{N L}^{*}=p_{P L}^{*}>p_{E L}^{*}, \lambda_{T}^{E}>\lambda_{T}^{P}=\lambda_{T}^{N}$ if $\tilde{p}_{T} \leq p^{1}$, and $p_{P L}^{*}>p_{N L}^{*}>p_{E L}^{*}, \lambda_{T}^{E}>\lambda_{T}^{P}>\lambda_{T}^{N}$ if $\tilde{p}_{T}>p^{1}$; when the regulation intensity is sufficiently loose $\left(\frac{\tau}{\alpha}<\beta<1\right), p_{P L}^{*}>p_{N L}^{*}>p_{E L}^{*}, \lambda_{T}^{E}>\lambda_{T}^{P}>\lambda_{T}^{N}$, where $p^{1}=\frac{\left(2 \alpha \bar{\alpha} V-c_{O L}(\alpha+1)\right)}{\alpha(\alpha-3)}$.

Proposition 3 shows that the entry limitation leads to the lowest price of low-end service and the highest demand of taxi service regardless of the regulation intensity and the taxi service price. Moreover, the price regulation can result in the price of low-end service and the demand of taxi service no less than that in the deregulation market. Different from the price regulation, the platform chooses a lower price strategy to deal with the entry limitation. Meanwhile, the reduction of price depends on the regulation intensity. To be specific, when the regulation intensity is sufficiently stringent and the price of taxi service is lower, the optimal platform's price with price regulation equals to that without regulation. However, the entry limitation leads to a lower price, which is beneficial to riders. Note that the entry limitation incurs a sharp slump in the demand of online car-hailing service, although the price is lower.

\section{Conclusion}

In this paper we explore the pricing decision of online car-hailing service with different regulation policies, i.e., price regulation and entry limitation. We compared the optimal platform's price and the demand of taxi service under these three scenarios: without regulation, with price regulation and with entry limitation. Our findings indicate that the price regulation increases the platform's price when the platform provides high-end service. Whereas the optimal platform's price of low-end service is the same with and without price regulation under certain circumstance. It is related to the taxi service price and the perceived service experience. The entry limitation reduces the optimal platform's price to achieve the regulation target. The result implies that entry limitation benefits not only riders but also taxi drivers. In this paper, we considered the platform as the decision maker; however, the online car-hailing service has the features of a twosided market. Drivers, self-employment workers, are entitled to decide when to work. Therefore, exploring the impact of new rules on performance of online car-hailing service based on two-sided market theory may be a possible direction for future research.

\section{Acknowledgement}

This work is supported by National Natural Science Foundation of China (NSFC) grant[grant number 72072125]. 


\section{References}

[1] Raju V. Implementing flexible systems in doctoral viva defense through virtual mechanism. Global Journal of Flexible Systems Management. 2021 Jun;22(2):127-39.

[2] Conway MW, Salon D, King DA. Trends in taxi use and the advent of ridehailing, 1995-2017: Evidence from the US National Household Travel Survey. Urban Science. 2018 Sep;2(3):79.

[3] Lavieri PS, Dias FF, Juri NR, Kuhr J, Bhat CR. A model of ridesourcing demand generation and distribution. Transportation Research Record. 2018 Dec;2672(46):31-40.

[4] Kortum K. New TRB special report: Between public and private mobility: Examining the rise of technology-enabled transportation services. TR News. $2016 \operatorname{Mar}(302)$.

[5] Pham A, Dacosta I, Jacot-Guillarmod B, Huguenin K, Hajar T, Tramèr F, Gligor V, Hubaux JP. Privateride: A privacy-enhanced ride-hailing service. Proceedings on Privacy Enhancing Technologies. 2017 Apr;2017(2):38-56.

[6] Marić J, Opazo-Basáez M. Green servitization for flexible and sustainable supply chain operations: A review of reverse logistics services in manufacturing. Global Journal of Flexible Systems Management. 2019 Dec;20(1):65-80.

[7] Zhong J, Lin Y, Yang S. The Impact of Ride-Hailing Services on Private Car Use in Urban Areas: An Examination in Chinese Cities. Journal of Advanced Transportation. 2020 Nov 23;2020.

[8] Deloitte. Market Analysis of Car-hailing Business. https://www2.deloitte.com/content/dam/Deloitte/cn/Documents/strategy/deloitte-cn-consulting-onlinecar-hailing-en-190221.pdf.2017.

[9] Jiang W, Zhang L. The impact of the transportation network companies on the taxi industry: Evidence from Beijing's GPS taxi trajectory data. Ieee Access. 2018 Feb 27;6:12438-50.

[10] Cetin T, Deakin E. Regulation of taxis and the rise of ridesharing. Transport Policy. 2019 Apr 1;76:149-58.

[11] Douglas GW. Price regulation and optimal service standards: The taxicab industry. Journal of Transport Economics and Policy. 1972 May 1:116-27.

[12] De Vany AS. Capacity utilization under alternative regulatory restraints: an analysis of taxi markets. Journal of political economy. $1975 \mathrm{Feb}$ 1;83(1):83-94.

[13] Sayarshad HR, Gao HO. A scalable non-myopic dynamic dial-a-ride and pricing problem for competitive on-demand mobility systems. Transportation Research Part C: Emerging Technologies. 2018 Jun 1;91:192-208.

[14] Wang J, Bai Z, Hu X. The effect of the integrated service mode and travel time uncertainty on taxis network equilibrium. Mathematical Problems in Engineering. 2015 Jan 1;2015.

[15] Yang H, Fung CS, Wong KI, Wong SC. Nonlinear pricing of taxi services. Transportation Research Part A: Policy and Practice. 2010 Jun 1;44(5):337-48.

[16] Wong RC, Yang L, Szeto WY, Li YC, Wong SC. The effects of accessible taxi service and taxi fare subsidy scheme on the elderly's willingness-to-travel. Transport Policy. 2020 Oct 1;97:129-36.

[17] Cachon GP, Daniels KM, Lobel R. The role of surge pricing on a service platform with self-scheduling capacity. Manufacturing \& Service Operations Management. 2017 Jul;19(3):368-84.

[18] Sun L, Teunter RH, Babai MZ, Hua G. Optimal pricing for ride-sourcing platforms. European Journal of Operational Research. 2019 Nov 1;278(3):783-95.

[19] Ma J, Xu M, Meng Q, Cheng L. Ridesharing user equilibrium problem under OD-based surge pricing strategy. Transportation Research Part B: Methodological. 2020 Apr 1;134:1-24.

[20] Bai J, So KC, Tang CS, Chen X, Wang H. Coordinating supply and demand on an on-demand service platform with impatient customers. Manufacturing \& Service Operations Management. 2019 Jul;21(3):556-70.

[21] Benjaafar S, Kong G, Li X, Courcoubetis C. Peer-to-peer product sharing: Implications for ownership, usage, and social welfare in the sharing economy. Management Science. 2019 Feb;65(2):477-93.

[22] Taylor TA. On-demand service platforms. Manufacturing \& Service Operations Management. 2018 Oct;20(4):704-20.

[23] Guda H, Subramanian U. Your uber is arriving: Managing on-demand workers through surge pricing, forecast communication, and worker incentives. Management Science. 2019 May;65(5):1995-2014.

[24] Furuhata M, Dessouky M, Ordóñez F, Brunet ME, Wang X, Koenig S. Ridesharing: The state-of-theart and future directions. Transportation Research Part B: Methodological. 2013 Nov 1;57:28-46.

[25] Benjaafar S, Hu M. Operations management in the age of the sharing economy: what is old and what is new?. Manufacturing \& Service Operations Management. 2020 Jan;22(1):93-101.

[26] Schroeter JR. A model of taxi service under fare structure and fleet size regulation. The Bell Journal of Economics. 1983 Apr 1:81-96.

[27] Harding S, Kandlikar M, Gulati S. Taxi apps, regulation, and the market for taxi journeys. Transportation Research Part A: Policy and Practice. 2016 Jun 1;88:15-25. 
[28] Bond AT. An app for that: Local governments and the rise of the sharing economy. Notre Dame L. Rev. Online. 2014;90:77.

[29] Wang S, Chen $\mathrm{H}$, Wu D. Regulating platform competition in two-sided markets under the $\mathrm{O} 2 \mathrm{O}$ era. International Journal of Production Economics. 2019 Sep 1;215:131-43.

[30] Yu JJ, Tang CS, Max Shen ZJ, Chen XM. A balancing act of regulating on-demand ride services. Management Science. 2020 Jul;66(7):2975-92.

[31] Szeto WY, Wong RC, Yang WH. Guiding vacant taxi drivers to demand locations by taxi-calling signals: A sequential binary logistic regression modeling approach and policy implications. Transport Policy. 2019 Apr 1;76:100-10.

[32] Gan J, An B. Game theoretic considerations for optimizing efficiency of taxi systems. InWorkshops at the Twenty-Ninth AAAI Conference on Artificial Intelligence 2015 Apr 1.

[33] Gan J, An B, Wang H, Sun X, Shi Z. Optimal pricing for improving efficiency of taxi systems. InTwenty-Third International Joint Conference on Artificial Intelligence 2013 Jun 30.

[34] Kim YJ, Hwang H. Incremental discount policy for taxi fare with price-sensitive demand. International Journal of Production Economics. 2008 Apr 1;112(2):895-902.

[35] Taylor BD, Chin R, Crotty M, Dill J, Hoel LA, Manville M, Polzin S, Schaller B, Shaheen S, Sperling D. Between Public and Private Mobility: Examining the Rise of Technology-Enabled transportation Services; Special Report 319. Transportation Research Board: Committee for Review of Innovative Urban Mobility Services: Washington, DC, USA. 2015.

[36] Jalloh, M. Uber: Advantages and Disadvantages. Investopedia. 2021.https://www.investopedia.com/articles/investing/110614/taxi-industry-pros-cons-uber-and-otherehail-apps.asp

[37] Camerer C, Babcock L, Loewenstein G, Thaler R. Labor supply of New York City cabdrivers: One day at a time. The Quarterly Journal of Economics. 1997 May 1;112(2):407-41.

[38] Allen EJ, Dechow PM, Pope DG, Wu G. Reference-dependent preferences: Evidence from marathon runners. Management Science. 2017 Jun;63(6):1657-72.

[39] Fehr E, Schmidt KM. A theory of fairness, competition, and cooperation. The quarterly journal of economics. 1999 Aug 1;114(3):817-68.

[40] Wei, X., Han, Z, Peng, C. The new policy of online car-hailing service is implemented for one year: it is difficult to "make a comeback" by taxi? Economic Information Daily. 2017. http://www.jjckb.cn/2017-07/28/c_136478886.htm

[41] Bellos I, Ferguson M, Toktay LB. The car sharing economy: Interaction of business model choice and product line design. Manufacturing \& Service Operations Management. 2017 May;19(2):185-201. 